3-9-2016

\title{
Ultrafast Multidimensional Laplace NMR Using a Single-Sided Magnet
}

Jared N. King

College of William and Mary

Vanessa J. Lee

College of William and Mary

Susanna Ahola

University of Oulu

Ville-Veikko Telkki

University of Oulu

Tyler Meldrum

College of William and Mary, tkmeldrum@wm.edu

Follow this and additional works at: https://scholarworks.wm.edu/aspubs

Part of the Chemistry Commons

\section{Recommended Citation}

J. N. King, V. J. Lee, S. Ahola, V.-V. Telkki, T. Meldrum, Angew. Chem. 2016, 128, 5124.

This Article is brought to you for free and open access by the Arts and Sciences at W\&M ScholarWorks. It has been accepted for inclusion in Arts \& Sciences Articles by an authorized administrator of W\&M ScholarWorks. For more information, please contact scholarworks@wm.edu. 


\title{
Ultrafast Multidimensional Laplace NMR Using a Single-sided Magnet
}

\author{
Jared N. King, ${ }^{[a]}$ Vanessa J. Lee, ${ }^{[a]}$ Susanna Ahola, ${ }^{[b]}$ Ville-Veikko Telkki, ${ }^{[b]}$ and Tyler Meldrum*[a]
}

\begin{abstract}
Laplace NMR (LNMR) consists of relaxation and diffusion measurements providing detailed information about molecular motion and interaction. In this communication, we demonstrate that ultrafast single- and multidimensional LNMR experiments, based on spatial encoding, are viable with low-field, single-sided magnets with an inhomogeneous magnetic field. This approach shortens the experiment time by one to two orders of magnitude relative to traditional experiments, and increases the sensitivity per unit time by a factor of three. The reduction of time required to collect multidimensional data opens significant prospects for mobile chemical analysis using NMR. Particularly tantalizing is future use of hyperpolarization to increase sensitivity by orders of magnitude, allowed by single-scan approach.
\end{abstract}

Nuclear magnetic resonance (NMR) spectroscopy is one of the most powerful and versatile tools in chemical analysis, ${ }^{[1]}$ and is widely exploited in medicine as magnetic resonance imaging (MRI). ${ }^{[2]}$ Single-sided magnets for NMR, first introduced over two decades ago, ${ }^{[3]}$ solve three major problems associated with traditional high-field NMR experiments: cost, immobility, and sample size restrictions. Single-sided hardware is roughly an order of magnitude less expensive than its high-field counterpart. Single-sided magnets' small size and low weight make them portable, and their open geometry allows for measurement of arbitrarily sized samples, including building materials, ${ }^{[4]}$ paintings and other objects in cultural heritage, ${ }^{[5]}$ and skin; ${ }^{[6]}$ they are also commonly used in well-logging. ${ }^{[7]}$

One major downside to single-sided magnets is that their magnetic fields are strongly inhomogeneous, preventing the observation of high-resolution NMR spectra. Impressive steps have been taken toward high-resolution spectra in inhomogeneous fields. ${ }^{[8]}$ However, despite their inhomogeneity, single-sided magnets still facilitate $T_{1}$ and $T_{2}$ relaxation as well as diffusion measurements. These measurements reveal details of molecular rotation and diffusion, explore interactions of nuclei with their microscopic environments, and can ultimately provide chemical resolution via these parameters. ${ }^{[1,3,9]}$ Relaxation and diffusion data consist of exponentially decaying components, and

[a] J. N. King, V. J. Lee, Prof. Dr. T. Meldrum

Department of Chemistry

The College of William \& Mary

P.O. Box 8795

Williamsburg, Virginia 23187-8795 (USA)

E-mail: tkmeldrum@wm.edu

http://www.wm.edu/chem/meldrum

[b] Dr. S. Ahola, Dr. V.-V. Telkki

NMR Research Group, Faculty of Science

University of Oulu

90014 Oulu (Finland) the distribution of diffusion coefficients or relaxation times can be extracted from the experimental data by an inverse Laplace transformation. ${ }^{[10]}$ Consequently, these methods are referred to as Laplace NMR (LNMR).

As with traditional NMR spectroscopy, the resolution and information content of LNMR can be enhanced by a multidimensional approach. ${ }^{[10,11]}$ Multidimensional LNMR deals with the correlation of relaxation times and/or diffusion coefficients with one another; it can also measure chemical exchange via these observables. This method has only recently entered routine use, following the development of a sufficiently reliable and robust multidimensional Laplace inversion algorithm in 2002. ${ }^{[12]}$ Multidimensional LNMR measurements can also be performed in inhomogeneous fields, including those produced by single-sided hardware. ${ }^{[5 b, 13]}$

Traditionally, multidimensional experiments increase the total measurement time, as the measurement has to be repeated with varying evolution delays. ${ }^{[1]}$ Long experiment times caused by these variable-delay repetitions generate several problems. Among these is the inability to use nuclear spin hyperpolarization techniques. Because generating hyperpolarization is typically very time-consuming (may take hours in some DNP setups) and different scans may receive different amounts of nuclear polarization, ${ }^{[14]}$ these methods that otherwise would increase the sensitivity by several orders of magnitude become inaccessible. However, recently it has been shown that $2 \mathrm{D}$ LNMR data can be measured in a single scan, ${ }^{[15]}$ based on continuous spatial encoding. This method of spatial encoding is similar to Frydman's ultrafast NMR spectroscopy ${ }^{[16]}$ and to single-scan 1D LNMR experiments. ${ }^{[17]}$ The ultrafast approach shortens the experiment time by one to three orders of magnitude, but at the cost of sensitivity (typically, sensitivity decreases by a factor of about four). However, the sensitivity per unit time is better in ultrafast experiments, because ultrafast measurements can be repeated many times in the same time that one traditional measurement requires. Furthermore, as the single-scan approach makes possible the use of hyperpolarized substances, overall sensitivity in the ultrafast experiment may potentially increase to many orders of magnitude higher than in conventional experiments. ${ }^{[15 b]}$

Single-sided magnets produce a magnetic field above the surface of the magnet that has an intrinsic, strong, and approximately constant (within the sensitive region of the RF coil) magnetic field gradient-this is in contrast with the pulsed gradients found in high-field experiments. ${ }^{[15,18]}$ In this communication, we demonstrate that the constant field gradient of single-sided magnets can be exploited to generate the spatial encoding and readout required for ultrafast multidimensional LNMR measurements. This encoding and readout scheme significantly shortens the experiment time and improves the sensitivity of low-field spectrometers. This demonstration is 
realized through the implementation of a $T_{1}-T_{2}$ correlation experiment.

The traditional[ ${ }^{[19]}$ and ultrafast $T_{1}-T_{2}$ pulse sequences for single-sided magnets are shown in Figure 1 ( $A$ and $B$ ); these sequences are similar to their high-field counterparts. ${ }^{[15 a, 19]}$ In the ultrafast pulse sequence, inversion-recovery-type $T_{1}$ encoding is performed with a frequency-swept CHIRP pulse, ${ }^{[17 a, 20]}$ followed by a $\pi / 2$ excitation pulse and subsequent $T_{2}$ encoding in the CarrPurcell-Meiboom-Gill (CPMG) ${ }^{[21]}$ loop. The adiabatic frequencyswept CHIRP pulse ${ }^{[22]}$ inverts the magnetization of the system, but because the frequency at which spins are inverted varies with time, and the Larmor frequency of the spins varies spatially due to the field gradient, different regions of the sample are inverted at different times (Figure 1C). With a CHIRP pulse of a duration that is a few times the $T_{1}$ of a sample, the first spins to be inverted will have largely returned to equilibrium magnetization before the $\pi / 2$ excitation pulse, while the last spins to be inverted will remain inverted. The recovery time of the magnetization can be obtained by the linear relationship between frequency, position, and time of inversion, thanks to the field gradient. Because the field gradient is present during acquisition, according to the principles of MRI, ${ }^{[2]}$ the Fourier transform of the first CPMG echo generates an inversion-recovery curve (Figures $1 \mathrm{D}$ and $\mathrm{E}$ ). The decay of the signal in subsequent CPMG echoes encodes $T_{2}$.

A)

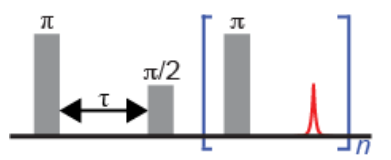

B)

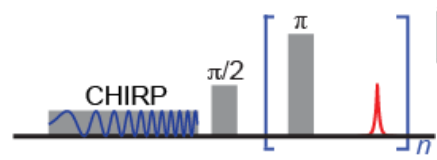

C)

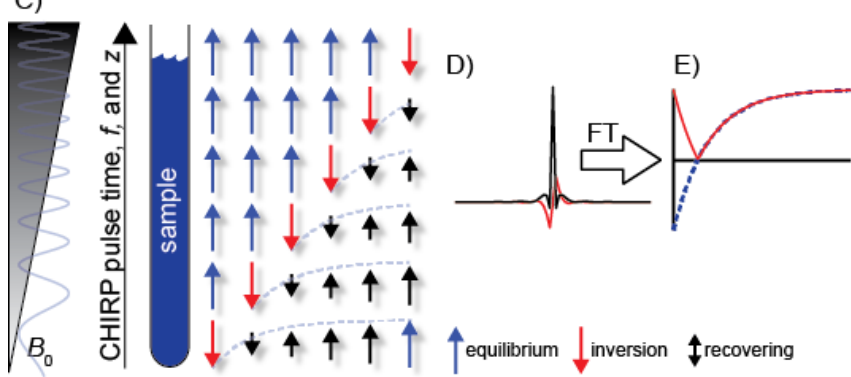

Figure 1. A) Traditional $T_{1}-T_{2}$ correlation sequence. The delay time $\tau$ is varied between scans (21 different values in these experiments) to construct the recovery of inverted magnetization, and the CPMG acquisition block, looped $n$ times, records transverse decay. B) Ultrafast $T_{1}-T_{2}$ correlation sequence. A CHIRP pulse with a linear frequency sweep is applied in lieu of a $\pi$-pulse. This CHIRP pulse, along with the magnetic field gradient, causes different regions of the sample to be inverted at different times, illustrated schematically in C). Each echo collected during the CPMG acquisition period encodes the spatially dependent inversion recovery information (shown in D), which can be recovered by Fourier transformation of the individual echoes $(\mathrm{E})$. Because the magnitude of the Fourier-transformed signal is obtained (solid red line in E), the user must select a point for manual inversion of the transformed data, producing a familiar inversion recovery curve (dashed blue line in $\mathrm{E}$ ). Since each echo encodes both inversion recovery and attenuation due to transverse relaxation, the data set can be used to construct a $T_{1}-T_{2}$ relaxation map.

Experimental ultrafast $T_{1}-T_{2}$ data of a gadolinium-doped water sample, measured with a single-sided magnet, is shown in Figure 2. The first column of the data and the coil excitation- detection profile (1D MR image of the sample, Figure 2A) reveal that the sensitive layer above the surface of the magnet is approximately $350 \mu \mathrm{m}$ in thickness, and the data is heavily weighted by the sensitivity profile. This weighting was compensated for by dividing the data by the coil excitationdetection profile. Furthermore, data points outside the CHIRP sweep region were removed and the sign of the magnitude data in the negative regions was changed (Figure 2B) before the $2 \mathrm{D}$ Laplace inversion. Importantly, extracting the inversion recovery record from the first echo and fitting it to an appropriate function provides a facile way of measuring $T_{1}$ only, without requiring a $2 \mathrm{D}$ Laplace inversion. This, then, represents the first demonstration of ultrafast measurements to record $T_{1}$ alone.

A)

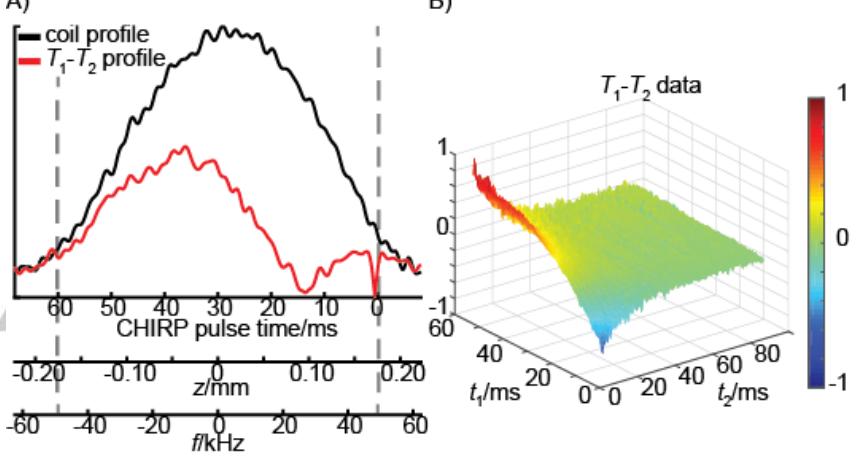

Figure 2. Ultrafast $T_{1}-T_{2}$ data of a glycerol sample following Fourier transformation. A) The first column of the 2D dataset, corresponding to the first inversion-recovery record, along with the coil excitation-detection profile. The CHIRP sweep region is indicated by vertical dashed lines, and the onset of the CHIRP sweep pulses causes the abrupt drop in signal at $0 \mathrm{~ms}$. B) The data after removing points outside the CHIRP sweep region, applying coil excitationdetection profile compensation, and changing the sign of the magnitude data in the negative regions. This dataset is then subjected to $2 \mathrm{D}$ inverse Laplace transformation to produce a $T_{1}-T_{2}$ correlation map.

Figure 3 shows $T_{1}-T_{2}$ maps of several samples, resulting from 2D Laplace inversions of data collected using both traditional and ultrafast methods. The maps include the same dominant peaks: the doped water and glycerol samples each produce one peak, while the double sample gives two peaks showing chemical resolution of the two compounds (water and glycerol). All the peaks have identical $T_{1}$ and $T_{2}$ values within experimental error in the traditional and ultrafast experiments (see Table 1), confirming the reliability of the ultrafast method. The maps include some additional artifacts, commonly encountered in the Laplace inversions of low signal-to-noise ratio (SNR) data. If the artifacts were to hinder the identification of the true peaks, one should measure the data with higher SNR or with slightly different experimental parameters; when this is done, the true peaks remain stationary while the artifacts change their position.

In addition to comparable accuracy, the speedup offered by the ultrafast method is remarkable. For example, in the doped water experiments with 1024 scans, the ultrafast experiment required only 5.6 minutes, compared with 116 minutes for the traditional experiment. On the other hand, spatial encoding lowers the sensitivity: the SNR for the conventional experiment is 1.21.6 times larger than in the ultrafast experiment (see Table 1). 
However, the ultrafast experiment can be repeated $N$ times (where $N$ is the number of indirect points in the traditional experiments) in the time of one traditional measurement, resulting in an $N^{1 / 2}$-fold increase in SNR. ${ }^{[1]}$ Consequently, the SNR per unit time is actually significantly higher in the ultrafast experiment than in the traditional: improvements of 2.9-3.7 times were observed in these experiments (see Table 1). In other terms, ultrafast experiments require approximately one-tenth the time of traditional experiments to achieve the same SNR. This improvement in sensitivity per unit time with a single-sided magnet is higher than at high field-at high field, the ultrafast improvement in sensitivity per unit time is about $1.8 .^{[15 \mathrm{~b}]}$ One reason for this may be the receiver bandwidth: in both traditional and ultrafast single-sided experiments, the bandwidth is the same because the magnetic field gradient is always present. In contrast, the receiver bandwidth in high-field experiments may be smaller in traditional experiments than in ultrafast ones, as there is no field gradient present during traditional experiments.
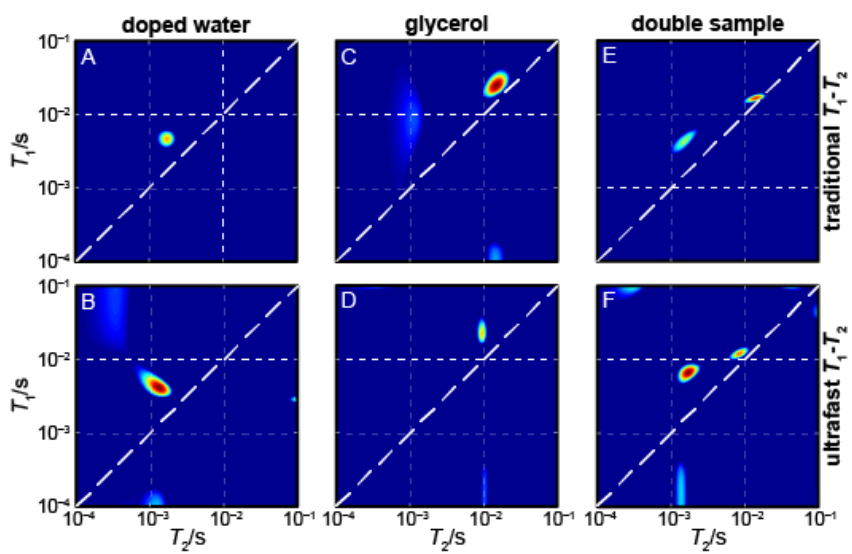

Figure 3. $T_{1}-T_{2}$ correlation maps obtained from 2D inverse Laplace transformation of the pre-processed data. Figures on the top row are from the traditional experiments, while the bottom row comes from the ultrafast experiments. A) and B) doped water; C) and D) glycerol; E) and F) a side-byside arrangement of glycerol and doped water. The $T_{1}$ and $T_{2}$ values obtained from these maps are given in Table 1. All figures come from measurements with 1024 scans; the diagonal dashed line in each indicates $T_{1}=T_{2}$.

The experimenter using ultrafast $T_{1}-T_{2}$ methods needs to be aware of some special features of the technique. The length of the CHIRP pulse needs to roughly match the $T_{1}$ of the sample. If the CHIRP pulse is longer than a few times $T_{1}$, much of the inverted magnetization will have recovered before the acquisition period, compromising the ability to determine small values of $T_{1}$. Conversely, if the CHIRP pulse is shorter than $T_{1}$, only the initial recovery of the magnetization is observed, leading to large uncertainties in $T_{1}$ values following inverse Laplace transformation. These restrictions necessitate some knowledge of $T_{1}$ beforehand, though this requirement is not substantively different from traditional procedures used to determine $T_{1}$.

In order to detect the magnetization profile accurately, the acquisition time in the CPMG loop has to be long enough, setting a lower limit for the echo time ( $700 \mu \mathrm{s}$ in the current experiments). This may restrict the observation of very short $T_{2}$ values.
The thickness of the magnetization encoding slab was about $350 \mu \mathrm{m}$ in the current experiments. The root-mean-square displacement of water molecules (diffusion coefficient about $2 \times 10^{-9} \mathrm{~m}^{2} \mathrm{~s}^{-1}$ ) during the CHIRP pulse (length $15-60 \mathrm{~ms}$ ) is about $10 \mu \mathrm{m}$; for glycerol it is much smaller. Consequently, the diffusional mixing of the spatial encoding is insignificant; this claim is supported by the agreement between the ultrafast and traditional experiments. Samples that have larger diffusion coefficients or that require longer CHIRP pulses may be more complex.

Table 1. $T_{1}, T_{2}$, and SNR values of the traditional (TRAD) and ultrafast (UF) $T_{1}-T_{2}$ experiments measured with 1024 scans. The number of indirect points in the traditional experiments was 21.

\begin{tabular}{|c|c|c|c|c|c|}
\hline sample & type & $T_{1} / \mathrm{ms}$ & $T_{2} / \mathrm{ms}$ & SNR & $\begin{array}{l}\text { SNR } \\
\text { increase }^{[a]}\end{array}$ \\
\hline doped water & TRAD & $5 \pm 1$ & $1.7 \pm 0.4$ & 181 & \\
\hline doped water & UF & $4 \pm 2$ & $1.3 \pm 0.5$ & 121 & 3.1 \\
\hline glycerol & TRAD & $24 \pm 10$ & $14 \pm 5$ & 84 & \\
\hline glycerol & UF & $22 \pm 9$ & $9 \pm 1$ & 54 & 2.9 \\
\hline $\begin{array}{l}\text { doped water + } \\
\text { glycerol }\end{array}$ & TRAD & $\begin{array}{l}4 \pm 1 \\
16 \pm 2\end{array}$ & $\begin{array}{l}1.5 \pm 0.5 \\
14 \pm 4\end{array}$ & 173 & \\
\hline $\begin{array}{l}\text { doped water + } \\
\text { glycerol }\end{array}$ & UF & $\begin{array}{l}7 \pm 1 \\
12 \pm 2\end{array}$ & $\begin{array}{l}1.7 \pm 0.5 \\
8 \pm 2\end{array}$ & 141 & 3.7 \\
\hline
\end{tabular}

[a] Improvement in SNR per unit time by the ultrafast method relative to the traditional one. SNR increase = scaled SNR(UF) / SNR(TRAD). Scaled SNR(UF) takes into account the fact that the UF experiment can be repeated 21 times in the experiment time of the traditional experiment, if the repetition rate is the same; scaled $\operatorname{SNR}(\mathrm{UF})=\operatorname{SNR}(\mathrm{UF}) \times(21)^{1 / 2}$.

The coil excitation-detection profile for single-sided instruments (Figure 2A) is very inhomogeneous. This inhomogeneity was compensated for by dividing the $T_{1}-T_{2}$ correlation data by the measured coil excitation-detection profile, as explained previously. The compensation should correct inhomogeneities in the first echo well, but the cumulative effects of $B_{1}$ inhomogeneities in subsequent $\pi$ pulses during the CPMG loop may lead to imperfect correction. On the other hand, multiple pulses in a CMPG train quickly compensate for imperfections in flip angle,$^{[21 b]}$ so this may not be a serious issue. This supposition is also supported by good experimental results.

The need for a sign change in the negative regions of the magnitude data in the inversion-recovery curve is one inconvenience of the current ultrafast $T_{1}-T_{2}$ method. This could be avoided by measuring phase-sensitive data or by supplanting the inversion-recovery encoding with saturation-recovery encoding, implemented with a $\pi / 2$ CHIRP pulse. ${ }^{[15 a]}$

In conclusion, this communication demonstrates that ultrafast LNMR experiments based on spatial encoding are viable with a low-field, single-sided magnet. They provide a remarkable time savings of one to two orders of magnitude, which can be converted to a significant (about three-fold) increase in SNR 
relative to traditional methods, while maintaining the same level of accuracy. Modifying other LNMR measurements, including molecular self-diffusion measurements, to be ultrafast experiments with single-sided magnets is a natural extension of this work. The single-scan nature of the ultrafast LNMR experiments also facilitates the use of hyperpolarized substances to increase sensitivity by several orders of magnitude. Currently, experiments require a large number of scans due to low thermal nuclear spin polarization at low field (about $10^{-6}$ ). However, if the polarization were increased to greater than 0.1 by nuclear spin hyperpolarization techniques, ${ }^{[14]}$ single-scan LNMR measurements would became feasible for samples with proton concentrations below $100 \mathrm{mM}$. This opens brilliant prospects for low-cost, mobile, real-time chemical analysis.

\section{Experimental Section}

Samples include water doped with $15 \mathrm{mM} \mathrm{GdCl}_{3}$ and (undoped) glycerol (Sigma-Aldrich, St. Louis, MO). First, the doped water and glycerol were measured individually, then put side-by-side in separate sample containers for a double sample. All data were collected using a singlesided PM25 magnet (Magritek, New Zealand) and Scout spectrometer (Tecmag, TX). The spectrometer operates at $13.24 \mathrm{MHz}$ and the magnet produces a field gradient of $6.59 \mathrm{~T} \mathrm{~m}^{-1}$. Hard $\pi$ and $\pi / 2$ pulses were calibrated to $6 \mu$ s with the power of the $\pi$ pulse twice that of the $\pi / 2$ pulse. The CHIRP pulses used in ultrafast experiments were calibrated to invert magnetization, with the power being dependent on the total length of the CHIRP pulse; in these experiments, the power of the $\pi$-CHIRP pulse was approximately one-tenth that of the hard $\pi$-pulse. The length of the CHIRP pulse was chosen to be on the order of the $T_{1}$ of the sample of interest, between 15 and $60 \mathrm{~ms}$. The echo time during the acquisition period was $700 \mu \mathrm{s}$, and a sufficient number of echoes was collected to observe the decay of magnetization to the level of noise ( 16 echoes for doped water, 64 echoes for the double sample, and 128 echoes for glycerol). A dwell time of $8 \mu$ s per complex point ( $125 \mathrm{kHz}$ bandwidth) was used and 76 complex points were collected per echo for an acquisition time of $608 \mu \mathrm{s}$ per echo. The number of accumulated scans was 1024 . A recovery period of $300-500 \mathrm{~ms}$ was appended to each scan. Traditional $T_{1}-T_{2}$ measurements were made using an inversion-recovery sequence with a CPMG detection scheme. ${ }^{[19]}$ The experimental parameters were similar to those used in the ultrafast experiments. However, to accommodate the grossly inhomogeneous field, the magnetization was inverted at the beginning of the sequence using a $50 \mu \mathrm{s}$, adiabatic iBURP pulse. ${ }^{[23]}$ Also, 21 points were collected in the indirect dimension for the inversion recovery (time delay $\tau$ ), ranging from $50 \mu$ s to $60 \mathrm{~ms}$; these points were linearly spaced.

Each echo collected in ultrafast experiments was zero-filled (one level) and Fourier transformed to produce a magnetization recovery curve. The recovery curves for all echoes were concatenated into a 2D dataset, which was then subjected (after removing the points outside the CHIRP sweep region and applying coil excitation-detection compensation) to an inverse Laplace transformation done in Prospa (Magritek). The traditional $T_{1}-T_{2}$ datasets were transformed similarly.

\section{Acknowledgements}

The financial support of the Academy of Finland (grant numbers 289649 and 294027) and the Emil Aaltonen Foundation is gratefully acknowledged.
Keywords: NMR spectroscopy • materials science • low-field • relaxation $\cdot$ Laplace

[1] J. Keeler, Understanding NMR spectroscopy, Wiley, Chichester, 2010.

[2] E. M. Haacke, R. W. Brown, M. R. Thompson, R. Venkatesan, Magnetic resonance imaging: Physical principles and sequence design, Wiley, New York, 1999.

[3] B. Blümich, J. Perlo, F. Casanova, Prog. Nucl. Magn. Res. Sp. 2008, 52, 197-269.

[4] a) A. Marko, B. Wolter, W. Arnold, J. Magn. Reson. 2007, 185, 19-25; b) B. Blümich, A. Haber, F. Casanova, E. Del Federico, V. Boardman, G. Wahl, A. Stilliano, L. Isolani, Anal. Bioanal. Chem. 2010, 397, 3117-3125.

[5] a) B. Blümich, F. Casanova, J. Perlo, F. Presciutti, C. Anselmi, B. Doherty, Acc. Chem. Res. 2010, 43, 761-770; b) V. Tudisca, C. Casieri, F. Demma, M. Diaz, L. Piñol, C. Terenzi, F. De Luca, J. Archaeol. Sci. 2011, 38, 352-359; c) D. Oligschläger, S. Waldow, A. Haber, W. Zia, B. Blümich, Magn. Reson. Chem. 2015, 53, 48-57; d) G. R. Fife, B. Stabik, A. E. Kelley, J. N. King, B. Blümich, R. Hoppenbrouwers, T. Meldrum, Magn. Reson. Chem. 2015, 53, 58-63.

[6] a) E. Ciampi, M. van Ginkel, P. J. McDonald, S. Pitts, E. Y. M. Bonnist, S. Singleton, A.-M. Williamson, NMR Biomed. 2011, 24, 135-144; b) E. Bergman, Y. Sarda, N. Ritz, E. Sabo, G. Navon, R. Bergman, U. Nevo, NMR Biomed. 2015, 28, 656-666.

[7] M. D. Hürlimann, D. D. Griffin, J. Magn. Reson. 2000, 143, 120-135.

[8] a) C.A. Meriles, D. Sakellariou, H. Heise, A.J. Moulé, A. Pines, Science 2001, 293, 82-85; b) B. Shapira, L. Frydman, J. Am. Chem. Soc. 2004, 126, 7184; c) J. Perlo, V. Demas, F. Casanova, C. A. Meriles, J. Reimer, A. Pines, B. Blümich, Science 2005, 308, 1279-1279; d) J. Perlo, F. Casanova, B. Blümich, Science 2007, 315, 1110-1112.

[9] a) D. E. Freed, L. Burcaw, Y.-Q. Song, Phys. Rev. Lett. 2005, 94, 067602; V.-V. Telkki, M. Yliniemi, J. Jokisaari, Holzforschung 2013, 67, 291-300.

[10] P. T. Callaghan, Translational Dynamics and Magnetic Resonance: Principles of Pulsed Gradient Spin Echo NMR, Oxford University Press, Oxford, 2011.

[11] a) Y.-Q. Song, J. Magn. Reson. 2013, 229, 12-24; b) D. Bernin, D. Topgaard, Curr. Opin. Colloid In. 2013, 18, 166-172.

[12] a) L. Venkataramanan, Y.-Q. Song, M. D. Hürlimann, IEEE Trans. Signal. Proc. 2002, 50, 1017-1026; b) Y.-Q. Song, L. Venkataramanan, M. D. Hürlimann, M. Flaum, P. Frulla, C. Straley, J. Magn. Reson. 2002, 154, 261-268.

[13] M. D. Hürlimann, L. Venkataramanan, J. Magn. Reson. 2002, 157, 3142.

[14] a) J. H. Ardenkjær-Larsen, B. Fridlund, A. Gram, G. Hansson, L. Hansson, M. H. Lerche, R. Servin, M. Thaning, K. Golman, Proc. Natl. Acad. Sci. USA 2003, 100, 10158-10163; b) S. R. Bowers in Encyclopedia of Nuclear Magnetic Resonance, Vol. 9 (Eds. D. M. Gant R. K. Harris), Wiley, Chichester, 2002, pp. 750-769; c) B. M. Goodson, J. Magn. Reson. 2002, 155, 157-216.

[15] a) S. Ahola, V.-V. Telkki, ChemPhysChem 2014, 15, 1687-1692; b) S Ahola, V. V. Zhivonitko, O. Mankinen, G. Zhang, A. M. Kantola, H.-Y Chen, C. Hilty, I. V. Koptyug, V.-V. Telkki, Nat. Commun. 2015, 6, 8363

[16] a) L. Frydman, T. Scherf, A. Lupulescu, Proc. Natl. Acad. Sci. USA 2002 99, 15858-15862; b) P. Pelupessy, J. Am. Chem. Soc. 2003, 125, 12345-12350; c) P. Giraudeau, L. Frydman, Annu. Rev. Anal. Chem. 2014, 7, 129-161.

[17] a) N. M. Loening, M. J. Thrippleton, J. Keeler, R. G. Griffin, J. Magn. Reson. 2003, 164, 321-328; b) M. J. Thrippleton, N. M. Loening, J. Keeler, Magn. Reson. Chem. 2003, 41, 441-447; c) P. E. S. Smith, K. J. Donovan, O. Szekely, M. Baias, L. Frydman, ChemPhysChem 2013, 14 , 3138-3145.

[18] J. Perlo, F. Casanova, B. Blümich, J. Magn. Reson. 2004, 166, 228-235.

[19] H. Peemoeller, R. K. Shenoy, M. M. Pintar, J. Magn. Reson. 1981, 45, 193-204. 
[20] R. L. Vold, J. S. Waugh, M. P. Klein, D. E. Phelps, J. Chem. Phys. 1968 48, 3831-3832.

[21] a) H. Y. Carr, E. M. Purcell, Phys. Rev. 1954, 94, 630-638; b) S. Meiboom, D. Gill, Rev. Sci. Instrum. 1958, 29, 688-691.
[22] M. Garwood, L. DelaBarre, J. Magn. Reson. 2001, 153, 155-177.

[23] H. Geen, R. Freeman, J. Magn. Reson. 1991, 93, 93-141. 
Entry for the Table of Contents (Please choose one layout)

Layout 1:

\section{COMMUNICATION}

Ultrafast experiments-one to two orders of magnitude faster than traditional experiments-measure multidimensional NMR relaxation parameters. Here, the ultrafast measurements are demonstrated using single-sided magnets, offering new possibilities for monitoring fast processes with few restrictions on sample size.

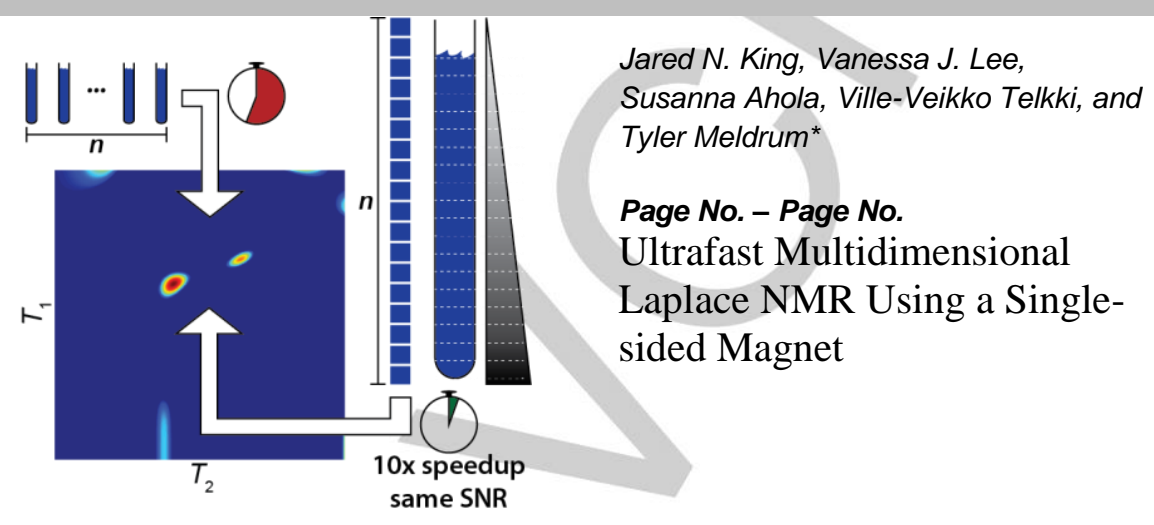

Layout 2:

COMMUNICATION

((Insert TOC Graphic here))
Author(s), Corresponding Author(s)*

Page No. - Page No.

Title

Text for Table of Contents 